\title{
Cooperative Vision in a Multi-Agent Architecture
}

\author{
Norbert Oswald and Paul Levi \\ University of Stuttgart, \\ Institute of Parallel and Distributed High-Performance Systems, \\ Applied Computer Science - Image Understanding, 70565 Stuttgart, Germany
}

\begin{abstract}
We present the concept of cooperative vision and its application to a multi-agent system with special attention to the integration of vision. Cooperative vision can be described as a type of distributed vision, where several agents working in a shared environment are involved. The object recognition task was distributed to several agents in order to demonstrate the concept of cooperative vision. This enables, on the one hand, a verification of objects by several agents and, on the other hand, a localization of spatial positions of other agents. A Bayesian approach is used for the combination of conclusions of several agents. Experiments done so far show significant results with regard to both tasks.
\end{abstract}

\section{Introduction}

Cooperative vision can be described as a type of distributed vision, where several agents working in a shared environment are involved. It can be sensible in a series of vision tasks but it requires arrangements for a local and temporal coordination between agents as well as strategies for the combination of individual conclusions. To achieve cooperative vision in a multi-agent system the design of an agent architecture has to meet certain criteria. One criterion is a modular concept for a multi-agent system that facilitates communication among agents, another is the design of a single component in form of a general frame to enable the integration of sensor perception and action. When vision is integrated into such an architecture, each kind of tight coupling of hardware components with control processes, as usually required in active vision applications, has to be bursted in order to avoid exclusive occupations of shared resources.

There are mainly two sorts of approach with regard to the design of agent architectures: The functional ones as suggested in [1] that support the planning aspects; and the behavior-based ones as in [2] that support reactivity. Attempts to combine both concepts were made by e.g. [6] and [4]. Crowley [4] developed a framework of how to build an active vision system that fulfils both low-level and higher-level tasks. The system aspect as a central role is also emphasized in [5]. There, use and integration of multiple cues and attention is described with an example of figure-ground segmentation. Dynamic attention and selective processing by static and dynamic belief nets is used for control in the VIEWS 
project [3]. To achieve computational efficiency in performing multiple visual tasks a tight coupling scheme is proposed. An early approach to an integration of multiple sources of information was proposed in the VISIONS project [7]. Previous research in the field of artificial intelligence has been related to bringing together uncertain data of several independent sources with Bayesian belief nets [10]. In [12], Bayesian nets were suggested to provide a general framework in visual tasks as control or decision making.

\section{A cooperative agent architecture}

In CoMRoS (Cooperative Mobile Robots Stuttgart) we use a matrix shaped agent architecture (fig. 1 (a)) as presented in detail in [11] [8]. Its modular design enables planning components, reactive behavior and cooperative task solving in a multi-agent environment.

The architecture is divided into three levels of abstraction, each containing a set of concurrent processes with equal rights. The strategical level is responsible for mission planning of agents in a wider range. The tactical level exercises contextual planning and execution control. It receives tasks from the strategical level, it plans solution strategies and supervises their execution. The reflexive level is composed of processes with a basically reactive behavior, some of them directly coupled to hardware components. Each of these processes has a sensoractuator coupling at one's disposal. This coupling represents an abstract control circuit that enables reactive behavior. We call these abstract control circuits autonomy cycles $(A C)$. Each $A C$ consists of units to decide, plan, learn, and several, parallel operating units to do and to monitor. Cooperation in the ar-

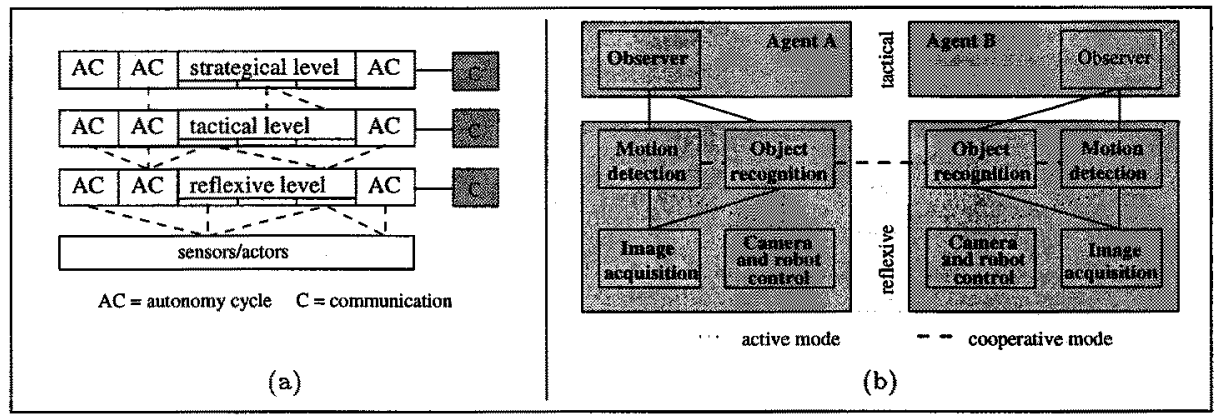

Fig. 1. Agent architecture (a) and integrated vision system with possible connection structures (b)

chitecture occurs, on the one hand, inside an agent between processes of the same level that can either be competitive, if accessing the same resources, or cooperative, if connected for data exchange, and thus requires strategies for conflict removal. On the other hand, cooperation occurs between multiple agents. 
Therefore, corresponding levels of the architecture are linked via dedicated communication modules to facilitate communication and arrangements.

\section{Integration of vision}

In our architecture each sensor type can be represented through a system that consists of several autonomy cycles on the reflexive and tactical level. A minimal vision system is currently embedded into the architecture as shown in fig. 1 (b). It is composed of an autonomy cycle on the tactical level, the Observer $\left(A C_{O b s}\right)$ that builds a behavior pattern to fulfil a given task, and four autonomy cycles on the reflexive level. The latter independently carry out elementary vision tasks such as motion detection, image recognition, respectively image acquisition, as well as camera and robot control.

Modes of the vision system. The actual connection structure within the behavior pattern between autonomy cycles on the reflexive level is given by the chosen plan of $A C_{O b s}$. This plan establishes whether autonomy cycles operate passive or active. Connection structures for autonomy cycles in active mode are shown by dotted lines in fig. 1 (b). While a camera control is not needed in passive mode, it is required in active mode to do well-directed camera movements. Only autonomy cycles that are not directly linked to any physical hardware components are able to operate in passive or active mode. In the vision system, this applies to the Motion detection $A C_{E m o}$ and to the Object recognition $A C_{S o r}$.

$A C_{E m o}$ pursues regions of motion in passive mode on the image plane without any camera movements. In active mode, $A C_{E m o}$ supplies a particular moving region to the camera cycle $A C_{C a m}$ for object tracking. In contrary to common active vision applications, the camera is not occupied exclusively. This facilitates a simultaneous usage of the camera or robot by any other autonomy cycle (e.g. $\left.A C_{S o r}\right) . A C_{S o r}$ recognizes objects according to [9] and operates in passive mode like a consumer. It works up all input but does not manipulate; it follows that the recognition method has to cope with arbitrary views. In active mode, $A C_{S o r}$ is able to influence the input data by choosing a particular view position. This action is supported by an appearance-based model database that additionally contains a description of how to move from one aspect to another. To guarantee an image from the new view position, the taking requires a synchronization between camera control and image acquisition.

Types of cooperation. Irrespective of the operation mode of autonomy cycles, the architecture enables two types of cooperation: internal and external. In the vision system, internal cooperation occurs in tasks where autonomy cycles $A C_{E m o}$ and $A C_{S o r}$ are linked as shown in fig. 1 (b) by broken lines. $A C_{E m o}$ is, on the one hand, used to simplify $A C_{S o r}$ by separating moving objects from background, on the other hand, to guarantee a continuous object recognition in image sequences by region identification. To select such a region of interest for 
$A C_{S o r}$, we currently use a selection algorithm as output filter in $A C_{E m o}$. This attention mechanism always tries to supply corresponding regions except the object recognition has found out that a particular region does not contain the object sought after. Though, as a consequence of internal cooperation, $A C_{S o r}$ can apply strategies to handle hypotheses that result from analysis of succeeding regions of motion in order to obtain more robust conclusions.

External cooperation in the vision system occurs in tasks where $A C_{S \text { or }}$ of several agents are connected. The aim of external cooperation is to solve a task with several agents working in a shared environment. In this way, an agent can exploit potentially better view positions of other agents for processsing. For cooperation, at first communication links between agents have to be built up to establish a work group at first. Therefore, we use a modified contract net protocol [13] between involved agents. The protocol is instantiated by any agent with request of support. Based on the established work group, external cooperation requires mechanisms to combine single evidences of involved agents to receive a common conclusion. A possible mechanism is presented subsequently.

\section{Aspects of cooperation}

In order to demonstrate the concept of cooperative vision we have distributed the recognition task to several agents. Following, we introduce feasible recognition tasks that appear in external cooperation. Then, a method for combining hypotheses of single runs is presented which can be used in internal or in external cooperation. Finally, we show how a distributed reasoning is done either by single or by continuous image analysis.

Cooperative recognition tasks. In general, we distinguish between two types of cooperative recognition tasks: verification and localization. The aim of the first task is to verify the object recognition result of a single agent by combining it with recognition results of all agents. In the second task, a suspected object is used to localize the relative spatial positions of other agents involved in the recognition. The basic pre-condition for cooperative identification is that agents observe the same environment. Geometrically this means, that the aperture angles of all cameras build a spatial section. To construct such a spatial section, the relative positions of all agents as well as their orientations of camera and robot have to be known respectively suspected. Fig. 2 (a) shows a spatial section between two cameras with a circle within and a square and triangle outside. In the verification task, known positions are assumed. Nevertheless, even in a spatial section agents have to make sure that they observe the same object. Objects like the circle not only have to be in the section but also must have been chosen from the attention mechanism. In the localization task, agents try to locate relative spatial positions of other agents according to a set of hypotheses. All hypotheses from two or more suspected viewpoints are combined by transformation to a reference viewpoint. The results are hypotheses about the orientation angle between two cameras. 


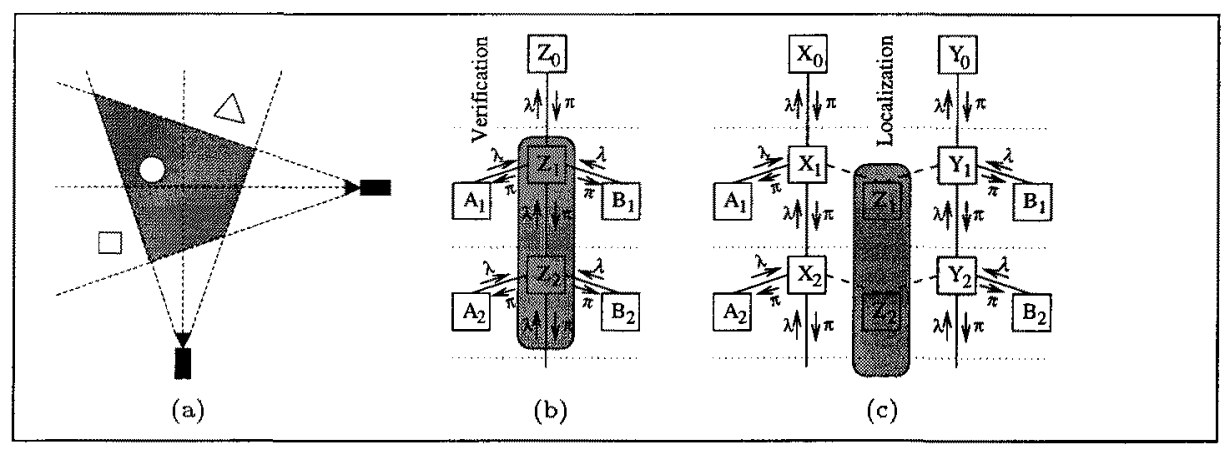

Fig. 2. Objects in 2 view fields (a), belief nets for verification (b) and localization (c)

Continuous recognition. With a continouos temporal analysis we avoid a strong rating of outliers from a single run and obtain assessments about objects and their orientation for each moment. Assume $A C_{S \text { or }}$ operates in active mode and observes a stationary unknown object. If the current maximum hypothesis about an object indicates the real aspect, then this hypothesis can be verified by a defined movement in the model space. Through such a movement that corresponds to a specific camera movement in the world a particular new aspect is expected to be rated strong in the next recognition run. The corresponding former aspect respectively hypothesis is verified, if the recognition algorithm will calculate a high energy value.

In general, a change from one aspect of an object to another occurs with a particular transition probability. Assumed, we have a prior knowledge about the transition probabilities as in the active mode at one's disposal, we can calculate predictions for the next observed aspects. Aspects $a_{i}$ and transition probabilities $r_{i, j}$ build an aspect transition matrix $T_{t_{k-1}, t_{k}}$ for each model at moments $t_{k-1}$ and $t_{k}$ of the following type:

\begin{tabular}{|c||c|c|c|c|c|}
\hline$t_{k-1} \backslash t_{k}$ & $a_{1}$ & $a_{2}$ & $a_{3}$ & $\ldots$ & $a_{n}$ \\
\hline$a_{1}$ & $r_{1,1}$ & $r_{1,2}$ & $r_{1,3}$ & $\ldots$ & $r_{1, n}$ \\
$a_{2}$ & $r_{2,1}$ & $r_{2,2}$ & $r_{2,3}$ & $\ldots$ & $r_{2, n}$ \\
$\ldots$ & $\ldots$ & $\ldots$ & $\ldots$ & $\ldots$ & $\ldots$ \\
$a_{n}$ & $r_{n, 1}$ & $r_{n, 2}$ & $r_{n, 3}$ & $\ldots$ & $r_{n, n}$ \\
\hline
\end{tabular}

Each row indicates the probability $r_{i, j}$, that aspect $a_{i}$ at time $t_{k-1}$ will change to $a_{j}$ at $t_{k}$. The sum of all $r_{i, j}$ for each row is supposed to be 1 . Like this, at time $t_{k}$ we expect a new set of hypotheses $h_{t_{k}^{\prime}}$ resulting from combination of the sets $h_{t_{k-1}}$ and $h_{t_{k}}$ with ratings $r_{i, j}$. In the continuous analysis, a different aspect transition matrix can be used at each time step. If we are absolutely certain about an aspect transition like in active mode, we set the corresponding $r_{i, j}$ to 1 and the remaining $r_{i, j}$ to 0 . In passive mode, a moving object has to be observed, otherwise there are no aspect transitions. Assuming a homogeneous motion of an unknown object, we know in advance that the new aspect is situated in an 
interval around the current aspect. Though, we set aspect ratings according to a distribution function, e.g. uniform or gaussian.

Combining several conclusions To combine resulting hypotheses, we use a Bayesian approach of information integration as proposed in [10]. In a cooperation protocol subsequent to the contract net, agents agree to a reference viewpoint to which all hypotheses are transformed and to a minimal parameter setting. In the verification task the known orientation angle between two cameras is used for the transformation of hypotheses. As shown in fig. 2 (b), the combination of results follows, with new evidences entering as diagnostic supports from camera $A$ in $A_{i}$ and from camera $B$ in $B_{i} . Z_{i}$ calculates new belief values for each diagnostic support together with the aspect transition matrices $T_{t_{i-1}, t_{2}}^{A_{i}}$ or $T_{t_{i-1}, t_{i}}^{B_{2}}$. The calculated belief values are simultaneously the new causal support $\pi$ to $Z_{i+1}$.

In the localization task, the orientation angle between two observers has to be determined. To be more robust, only values from a continuous analysis are considered for combination (fig. 2 (c)). From that we get two belief nets $X$ and $Y$ for camera $A$ and $B$. One camera is chosen to be the reference viewpoint with the reference set of hypotheses. Hypotheses of the other camera are transformed by any orientation angle. Then the belief net combines both sets as in the verification task and calculates belief values for $Z_{i}$ which denote aspects. The combination with the reference set is done for varying orientation angles. The number of possible orientation angles depends on the current model set of aspects. As a result from that, the maximum belief value represents an estimation about the suspected orientation angle.

Experimental results To present (the first) experimental results for both cooperative recognition tasks, we observed an unknown moving object with two agents operating in passive mode. Fig. 3 (a) shows a part of that image sequence where the object was mutually observed by the two agents. The orientation angle between the cameras of agent $\mathrm{A}$ and $\mathrm{B}$ was approximately $120^{\circ}$. The used model set contained 24 aspects of our robot in $15^{\circ}$ steps from $0^{\circ}$ to $360^{\circ}$ taken from a fixed distance. All aspect transition matrices were filled according to a uniform distribution in the interval $[i-1, i+1]$ around an aspect $a_{i}$.

In the verification task, object analysis and the combination of resulting hypotheses was performed in a continuous belief net according to fig. 2 (b). The camera position of agent $B$ was chosen as a reference viewpoint. In fig. 3 (b), the horizontal axis shows time steps $t_{i}$ of the image sequence, the vertical axis describes the suspected aspect in degree. We see a comparison between continuous and cooperative analysis in relation to the approximated real object aspect over a time interval. Each graph shows aspects corresponding to the calculated maximum hypothesis for each time step. Of course, all hypotheses depend on results of single runs which, in turn, depend on the quality of the segmentation. Observer $B$ has a more homogeneous background than observer $A$ and distortion that influences the object recognition is mostly compensated. 


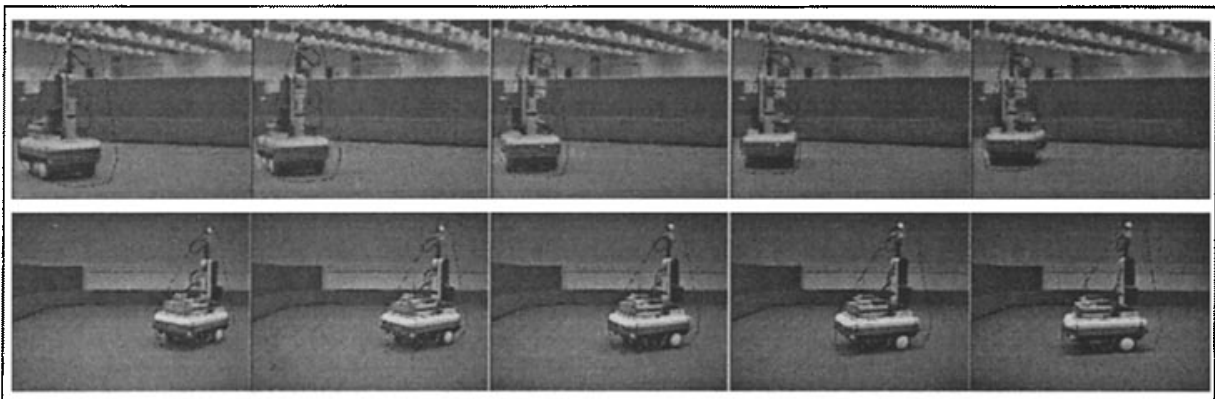

(a)

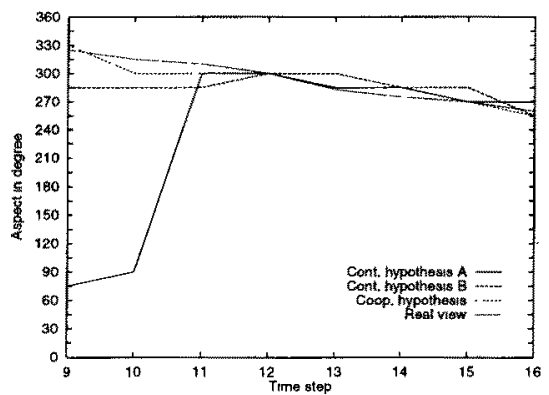

(b)

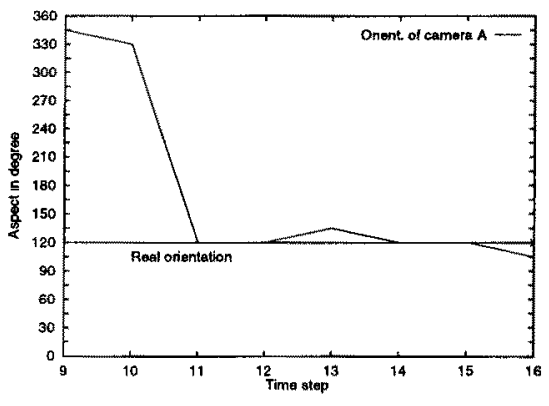

(c)

Fig. 3. Image sequence of a scene observed from two view points (a), calculated aspects of an object observed by two agents and projected to the reference viewpoint of camera $B$ for each time step $\Delta t(b)$, estimated orientation of camera $A$ as a result of object analysis from the reference viewpoint of camera B (c).

Thus, its propositions from single runs about the suspected object aspect is approximately correct. In contrast to that, observer $A$ has to cope with changes in illumination because the extracted region of interest from $A C_{E m o}$ contains neon strips. Thus predictions vary, although the convex hull has correctly been cut. From that, camera $A$ needs three time steps to adapt the real object aspect. A combination of recognition results from camera $A$ and $B$ leads to a quick and good approximation of the real object aspect. That means, that agent $A$ gets fairly accurate hypotheses at each time step.

In the localization task, agents combine both sets of hypotheses continuously to calculate for each time step an estimation about the current orientation angle (fig. 2 (c)). The angle is determined by calculating the maximum hypothesis for each possible orientation related to viewpoint $B$. The combination (fig. 3 (c)) supplies a fairly good evidence for the actual orientation angle. The accuracy of any result depends on the aspect difference in the model set. If segmentation supplies poor input, the recognition results will not be very accurate. But as it was demonstrated above with agent $A$, if at least one agent is able to calculate correct recognition results, other agents that deal with poor input will profit. 


\section{Conclusion}

We presented the concept of cooperative vision applied to a multi-agent system with an example of a distributed recognition task. In the proposed architecture, a vision system is integrated as a system of loosely coupled autonomy cycles which facilitates interlaces with autonomy cycles that belong to other sensor types. The architecture makes possible two types of cooperation, on the one hand, internal cooperation that appears inside an agent, on the other hand, external cooperation that appears between several agents. To combine calculated conclusions of several agents to single evidences we used belief nets. This mechanism is applied to the verification and localization task, where objects are verified and combined by several agents in order to get more robust recognition result. Results show that agents can profit through a common recognition, especially when the quality of its own input is poor. The localization task facilitates the determination of local spatial positions of other agents based on individual recognition results. Experiments show a fairly good approximation of the actual relative orientation.

\section{References}

1. Albus, J.S., McCain, H.G., Lumia, R.: NASA/ NBS Standard Reference Model for Telerobot Control System Architecture. Technical Note 1235 (1987)

2. Brooks, R.A.: A Layered Control System for a Mobile Robot. 3rd Symposium. MIT Press (1986) 367-372

3. Buxton, H., Gong, S.: Advanced Visual Surveillancce using Bayesian Networks. AI-Journal (1997) (to appear)

4. Crowley, J. et al.: Integration and Control of Reactive Visual Processes. Lecture Notes in Computer Science 801 (1994) 47-58

5. Eklundh, J.-O., Nordlund, P., Uhlin, T.: Issues in active vision: attention and cue integration/ selection. BMVC (1996)

6. Fleury S., Herrb M., Chatila R.: Design of a Modular Architecture for Autonomous Robot. IEEE Robotics and Automation (1994) 3508-3513

7. Hanson, A.R., Riseman, E.M.: The VISIONS Image Understanding System - 1986. COINS Technical Report 86-92 (1986)

8. Levi, P.: Architectures of individual and distributed autonomous agents. IAS-2 (1989) 315-324

9. Oswald, N., Gerl, S., Biedert, R.: Konfigurationsbasiertes Verfahren zur schnellen Identifikation komplexer Objekte. DAGM (1996) 187-195

10. Pearl, J.: Distributed Revision of Composite Beliefs. Artificial Intelligence 33 (1987) 173-215

11. Rausch, A., Oswald, N.: Cooperative Crossing of traffic intersections in a distributed robot system. SPIE 2589 (1995) 218-229

12. Rimey, R.D.: Control of Selective Perception Using Bayes Nets and Decision Theory. University of Rochester. Technical Report 468 (1993)

13. Smith, R.G.: The Contract Net Protocol: High-Level Communication and Control in a Distributed Problem Solver. IEEE Transactions on Computers C-29 No. 12 (1980) 1104-1113 